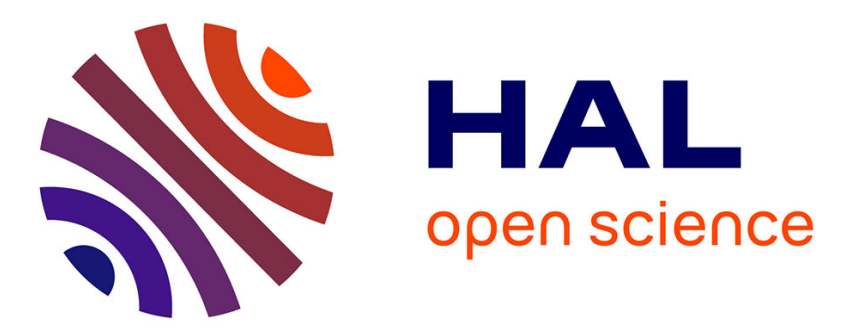

\title{
Onset of collectivity in neutron-rich Fe isotopes: Toward a new island of inversion?
}

J. Ljungvall, A. Gorgen, A. Obertelli, W. Korten, E. Clément, G. de France, A. Burger, J.-P. Delaroche, A. Dewald, A. Gadea, et al.

\section{- To cite this version:}

J. Ljungvall, A. Gorgen, A. Obertelli, W. Korten, E. Clément, et al.. Onset of collectivity in neutronrich Fe isotopes: Toward a new island of inversion?. Physical Review C, 2010, 81, pp.061301. 10.1103/PhysRevC.81.061301 . in2p3-00495174

\section{HAL Id: in2p3-00495174 https://hal.in2p3.fr/in2p3-00495174}

Submitted on 2 Dec 2010

HAL is a multi-disciplinary open access archive for the deposit and dissemination of scientific research documents, whether they are published or not. The documents may come from teaching and research institutions in France or abroad, or from public or private research centers.
L'archive ouverte pluridisciplinaire HAL, est destinée au dépôt et à la diffusion de documents scientifiques de niveau recherche, publiés ou non, émanant des établissements d'enseignement et de recherche français ou étrangers, des laboratoires publics ou privés. 


\title{
Onset of collectivity in neutron-rich Fe isotopes: Toward a new island of inversion?
}

\author{
J. Ljungvall,,${ }^{1,2,3}$ A. Görgen, ${ }^{1}$ A. Obertelli, ${ }^{1}$ W. Korten, ${ }^{1}$ E. Clément,${ }^{2}$ G. de France ${ }^{2}$ A. Bürger, ${ }^{4}$ \\ J.-P. Delaroche ${ }^{5}$ A. Dewald,${ }^{6}$ A. Gadea, ${ }^{7}$ L. Gaudefroy, ${ }^{5}$ M. Girod, ${ }^{5}$ M. Hackstein, ${ }^{6}$ J. Libert ${ }^{8}$ \\ D. Mengoni, ${ }^{9}$ F. Nowacki, ${ }^{10}$ T. Pissulla, ${ }^{6}$ A. Poves, ${ }^{11}$ F. Recchia, ${ }^{12}$ M. Rejmund, ${ }^{2}$ W. Rother,${ }^{6}$ E. Sahin, ${ }^{12}$ \\ C. Schmitt ${ }^{2}$ A. Shrivastava ${ }^{2}$ K. Sieja, ${ }^{10}$ J.J. Valiente-Dobón, ${ }^{12}$ K.O. Zell, ${ }^{6}$ and M. Zielińska ${ }^{13}$ \\ ${ }^{1}$ CEA Saclay, IRFU, Service de Physique Nucléaire, F-91191 Gif-sur-Yvette, France \\ ${ }^{2}$ GANIL, CEA/DSM-CNRS/IN2P3, Bd Henri Becquerel, BP 55027, F-14076 Caen, France \\ ${ }^{3}$ CSNSM, CNRS/IN2P3, F-91405 Orsay, France \\ ${ }^{4}$ Department of Physics, University of Oslo, PO Box 1048 Blindern, N-0316 Oslo, Norway \\ ${ }^{5}$ CEA, DAM, DIF, F-91297 Arpajon, France \\ ${ }^{6}$ Institut für Kernphysik, Universität zu Köln, D-50937 Köln, Germany \\ ${ }^{7}$ Instituto de Fisica Corpuscular, CSIC-Universidad de Valencia, E-46071 Valencia, Spain \\ ${ }^{8}$ Institut de Physique Nucléaire, CNRS/IN2P3-Université Paris-Sud, F-91406 Orsay, France \\ ${ }^{9}$ Dipartimento di Fisica dell'Università and INFN Sezione di Padova, I-35131 Padova, Italy \\ ${ }^{10}$ IPHC, CNRS/IN2P3 and Université Louis Pasteur, F-67037 Strasbourg, France \\ ${ }^{11}$ Departamento de Física Teórica, IFT-AM/CSIC, Universidad Autónoma, E-28049 Madrid, Spain \\ ${ }^{12}$ INFN, Laboratori Nazionali di Legnaro, I-35020 Legnaro, Italy \\ ${ }^{13}$ Heavy Ion Laboratory, Warsaw University, Warsaw, PL-02097, Poland
}

(Dated: May 11, 2010)

\begin{abstract}
The lifetimes of the first excited $2^{+}$states in ${ }^{62} \mathrm{Fe}$ and ${ }^{64} \mathrm{Fe}$ have been measured for the first time using the recoil-distance Doppler shift method after multi-nucleon transfer reactions in inverse kinematics. A sudden increase of collectivity from ${ }^{62} \mathrm{Fe}$ to ${ }^{64} \mathrm{Fe}$ is observed. The experimental results are compared with new large-scale shell model calculations and Hartree-Fock-Bogolyubovbased configuration-mixing calculations using the Gogny D1S interaction. The results give a deeper understanding of the mechanism leading to an onset of collectivity near ${ }^{68} \mathrm{Ni}$, which is compared with the situation in the so-called island of inversion around ${ }^{32} \mathrm{Mg}$.
\end{abstract}

PACS numbers: 21.10.Re Collective levels 21.10.Tg Lifetimes 27.50.+e $59 \leq A \leq 89$

It is a common feature of systems of interacting fermions to form a shell structure. In atomic nuclei the spin-orbit interaction lowers the energy of the orbitals with highest angular momentum into the next lower oscillator shell with opposite parity, leading to the wellknown sequence of magic numbers. While the shell structure and the resulting energy gaps between the orbitals explain many general properties of nuclei across the nuclear chart, it has become evident that the shell structure and magic numbers change for nuclei with large neutron excess. As protons and neutrons in such exotic nuclei occupy different orbitals compared to their stable counterparts, the effective single-particle energies are shifted, so that some shell gaps vanish and new ones emerge [1]. It is important to find experimental signatures for the changing shell structure in order to advance the theoretical description of exotic nuclei, e.g. by finding better shell model interactions or better energy functionals for mean-field based models.

The region of neutron-rich nuclei between $Z=20$ (Ca) and $Z=28(\mathrm{Ni})$ is of particular interest for understanding the evolution of the shell structure for nuclei with large neutron excess. Adding protons to the $1 f_{7 / 2}$ orbital changes the relative energies of the neutron $2 p_{3 / 2}$, $2 p_{1 / 2}$, and $1 f_{5 / 2}$ orbitals due to the strongly attractive proton-neutron spin-flip interaction [2]. With the gradual filling of the neutron $f p$ harmonic oscillator shell, excitations into the $1 g_{9 / 2}$ intruder orbital become increasingly important. Subtle effects related to the energy gap between the $f p$ shell and the $1 g_{9 / 2}$ orbital lead to a strong variation of collectivity for nuclei with $N \approx 40$. The nucleus ${ }_{28}^{68} \mathrm{Ni}_{40}$ has many features of a doubly-magic nucleus with high excitation energy of the $2_{1}^{+}$state [3] and a small $B\left(E 2 ; 0_{1}^{+} \rightarrow 2_{1}^{+}\right)$value [4]. Mass measurements, on the other hand, show that the $N=40$ gap is weak for ${ }^{68} \mathrm{Ni}[5,6]$, and it has been argued that the small $B(E 2)$ value does not necessarily indicate a shell gap at $N=40$, but that it reflects the character of the $2_{1}^{+}$state as a predominant neutron excitation [7]. The fragility of the $N=40$ sub-shell gap is further evidenced by the developing collectivity in the neutron-rich Zn and Ge isotopes, for which the energy of the $2_{1}^{+}$state drops sharply from $N=38$ to $N=42$ while the $B(E 2)$ values increase $[8,9]$. The removal of protons from the $f_{7 / 2}$ orbital has a similar effect. The $2_{1}^{+}$energies in the Fe isotopes drop sharply above $N=36$. The removal of only two protons changes the energy of the $2_{1}^{+}$state from $2033 \mathrm{keV}$ in ${ }^{68} \mathrm{Ni}$ to $573 \mathrm{keV}$ in ${ }^{66} \mathrm{Fe}$ [10]. The $2_{1}^{+}$energy drops even further to $517 \mathrm{keV}$ in ${ }^{68} \mathrm{Fe}$ [11]. A similar behavior is observed in the $\mathrm{Cr}$ isotopes, where the $2_{1}^{+}$energies decrease gradually beyond the $N=32$ sub-shell closure [12]. Inelastic proton scattering experiments [13] and shell model studies [14] corroborate the development of substantial collectivity and deformation in the neutron-rich $\mathrm{Cr}$ isotopes.

The role of the $\nu g_{9 / 2}$ orbital for the onset of collectivity in the region below ${ }^{68} \mathrm{Ni}$ has been discussed contro- 
versially in the past. The excitation spectrum of ${ }^{64} \mathrm{Fe}$ was established by Hoteling el al. and compared to shell model calculations restricted to the $f p$ model space [15]. Since the calculations reproduced the energy of the $2^{+}$ state reasonably well, it was concluded that the $\nu g_{9 / 2}$ orbital is not responsible for the low $2_{1}^{+}$energy and that it becomes important only at higher energies, where the calculations could not reproduce the observed states. Lunardi et al. have studied neutron-rich Fe isotopes of both even and odd mass [16]. Comparing the results to shell model calculations in the $f p g$ model space it was concluded that the occupation of the $g_{9 / 2}$ orbital influences the structure of the neutron-rich Fe isotopes considerably. The increasing role of the $g_{9 / 2}$ orbital is also consistent with the decreasing excitation energy of $9 / 2^{+}$isomers in odd-mass Fe nuclei [17]. In studies of the odd-mass Mn isotopes, Valiente-Dobón et al. have argued that including the effects of the $g_{9 / 2}$ orbital improves the shell model description of ${ }^{61} \mathrm{Mn}_{36}$ and ${ }^{63} \mathrm{Mn}_{38}$ [18], while Crawford et al. have interpreted the level structure in ${ }^{61} \mathrm{Mn}$ with a low level density below $1 \mathrm{MeV}$ without the need to invoke neutron excitations into the $g_{9 / 2}$ orbital [19]. In the $\mathrm{Cr}$ isotopes there is clear evidence for the influence of the $g_{9 / 2}$ orbital already for ${ }^{59} \mathrm{Cr}_{35}$ [20].

A deeper understanding of how collectivity develops in this region of the nuclear chart can come from experimental $B(E 2)$ values. For the even-even nuclei $B(E 2)$ values have been measured up to ${ }^{58} \mathrm{Cr}_{34}[21],{ }^{60} \mathrm{Fe}_{34}[22]$, and ${ }^{70} \mathrm{Ni}_{40}[23]$. Here we report on a lifetime measurement for the $2_{1}^{+}$states in ${ }^{62} \mathrm{Fe}$ and ${ }^{64} \mathrm{Fe}$ performed at the Grand Accelerateur National d'Ions Lourds using the recoil-distance Doppler shift (RDDS) method [24] in combination with multi-nucleon transfer reactions in inverse kinematics. The technique is similar to the one used in a recent lifetime measurement in ${ }^{50} \mathrm{Ca}$ and ${ }^{51} \mathrm{Sc}$ in direct kinematics at Legnaro [25]. Excited states in a wide range of neutron-rich nuclei were populated in multinucleon transfer reactions between a ${ }^{238} \mathrm{U}$ beam at $6.5 \mathrm{~A}$ $\mathrm{MeV}$ and a ${ }^{64} \mathrm{Ni}$ target of $1.5 \mathrm{mg} / \mathrm{cm}^{2}$ thickness. The target-like reaction products were detected and identified event by event in the large-acceptance spectrometer VAMOS [26]. The optical axis of the spectrometer was positioned at $45^{\circ}$ with respect to the beam axis, close to the grazing angle of the reaction. The focal plane detection system of the spectrometer allows the full reconstruction of the trajectories through the spectrometer and an unambiguous identification of the reaction products in mass, charge, and atomic number (see Fig. 1). The magnetic rigidity of the spectrometer was optimized for the transmission of ${ }^{64} \mathrm{Fe}$.

The target was surrounded by nine large, segmented germanium clover detectors of the Exogam array [27], one at $180^{\circ}$, three at $135^{\circ}$, and five at $90^{\circ}$ relative to the spectrometer axis. The typical average recoil velocity of the target-like reaction products exiting the target foil was $37 \mu \mathrm{m} / \mathrm{ps}$. In order to apply the RDDS technique the velocity of the recoils was slowed to approximately $32 \mu \mathrm{m} / \mathrm{ps}$ in a $4.7 \mathrm{mg} / \mathrm{cm}^{2}$ thick magnesium foil

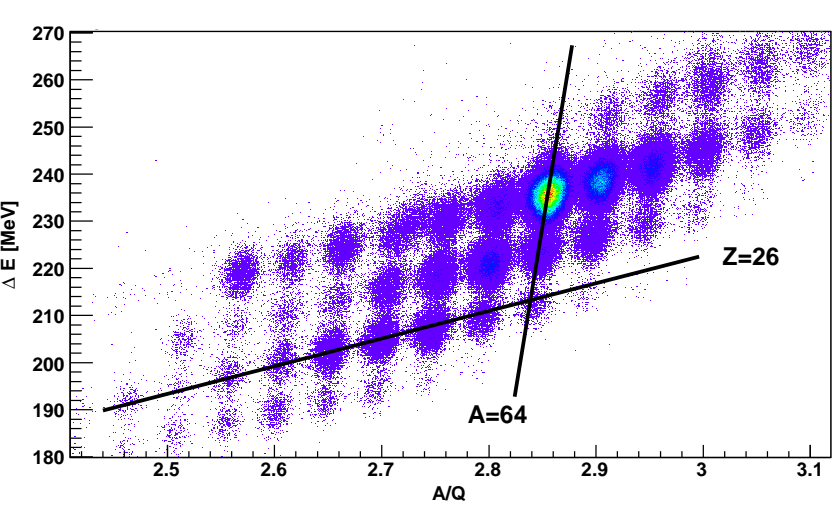

FIG. 1: Typical identification plot showing the energy loss of the ions in the ionization chamber of VAMOS as a function of the mass-over-charge ratio, which is determined from the time of flight through the spectrometer and the magnetic rigidity. The lines indicate the loci of ions with $A=64$ and $Z=26$, respectively. The most intense peak corresponds to elastically scattered ${ }^{64} \mathrm{Ni}$ target nuclei. The data is shown for only one of 21 silicon detectors mounted in VAMOS and for one single charge state $(Q=23)$. The identification of the ions was performed in the same way for all detectors and all charge states reaching the focal plane.

placed at micrometer distances behind the target. The different Doppler shifts of $\gamma$ rays emitted before and after the degrader foil were measured in the germanium detectors under backward angles. The distance between target and degrader was adjusted and controlled by a compact plunger device [28]. Data was collected for six distances between 40 and $750 \mu \mathrm{m}$. An additional measurement was performed without degrader to determine the exact recoil velocities after the target.

The spectra of Fig. 2 show $\gamma$ rays recorded in coincidence with ions identified as ${ }^{62} \mathrm{Fe}$ and ${ }^{64} \mathrm{Fe}$ for selected target-degrader distances. A Doppler correction was performed using the recoil velocity measured in VAMOS after the degrader foil and the relative angle between the $\gamma$ ray and the recoil vector. Two peaks are observed for the $2_{1}^{+} \rightarrow 0_{1}^{+}$transitions in both ${ }^{62} \mathrm{Fe}$ and ${ }^{64} \mathrm{Fe}$, corresponding to the different recoil velocities before and after the degrader foil, respectively. The peaks are well separated for relative angles greater than $135^{\circ}$. Spectra were produced and analyzed separately for two angular ranges between $135^{\circ}$ and $150^{\circ}$ and between $160^{\circ}$ and $180^{\circ}$, respectively. Decay curves $I_{d} /\left(I_{d}+I_{f}\right)$ were constructed from the intensities of the degraded $\left(I_{d}\right)$ and fully Doppler shifted $\left(I_{f}\right)$ components of the transitions as a function of target-degrader distance. The normalization factors $\left(I_{d}+I_{f}\right)$ were found to be consistent with the number of ions identified in VAMOS for each distance. Lifetimes of the $2_{1}^{+}$states in ${ }^{60} \mathrm{Fe},{ }^{62} \mathrm{Fe}$, and ${ }^{64} \mathrm{Fe}$ were extracted separately for the two angular ranges from the decay curves of the $2_{1}^{+}$and $4_{1}^{+}$states using the differential decay curve (DDC) method [24] and then combined into a weighted average. In the DDC method the lifetime of a state is determined for each target-degrader distance from the 
and $d_{5 / 2}$ orbitals increase to 2.2 and 0.72 , respectively, which drives the nucleus to an intrinsically deformed prolate shape (with $\beta \approx 0.35$ ) and causes a more rotational yrast sequence consistent with experimental observations [13]. The transition from spherical ${ }^{68} \mathrm{Ni}$ to more proton deficient $N \approx 40$ isotones with deformed intruder configurations seems to have some similarity to the situation in the so-called island of inversion of $\mathrm{Na}$ and $\mathrm{Mg}$ isotopes with $N \approx 20$ (see e.g. Ref. [1] for a review). In both cases the developing quadrupole collectivity can be related to the occupation of neutron intruder orbitals from the next oscillator shell, which are at the same time quasi-SU(3) partners: $\left(f_{7 / 2}, p_{3 / 2}\right)$ for ${ }^{32} \mathrm{Mg}$ and $\left(g_{9 / 2}, d_{5 / 2}\right)$ in the present case. Active quadrupole partners are also available for the protons: $\left(d_{5 / 2}, s_{1 / 2}\right)$ for $\mathrm{Mg}$ and $\left(f_{7 / 2}, p_{3 / 2}\right)$ for Fe and Cr. The shell model calculations furthermore find a reduction in the harmonic oscillator gap at $N=40$ from ${ }^{68} \mathrm{Ni}$ to ${ }^{60} \mathrm{Ca}$. The sudden increase of collectivity from ${ }^{62} \mathrm{Fe}$ to ${ }^{64} \mathrm{Fe}$ can thus be understood in a similar way as the increase of collectivity from ${ }^{30} \mathrm{Mg}$ to ${ }^{32} \mathrm{Mg}$.

It is clear, on the other hand, that there is no shell closure at $N=40$ comparable to the one at $N=20$. At this point the analogy between the island of inversion, which is situated at the end of a long chain of spherical $N=20$ isotones, and the $N=40$ region breaks down. With the exception of ${ }^{68} \mathrm{Ni}$ with closed proton shell, all $N=40$ isotones develop collectivity and deformation (see left-hand part of Fig. 3). In a recent study within a microscopic collective model, the low-lying states in the $N=40$ isotones (except ${ }^{68} \mathrm{Ni}$ ) were described as prolate deformed ground-state bands and quasi- $\gamma$ bands with good overall agreement between the calculations and experimental data [34]. We have extended these studies along the chain of $\mathrm{Fe}$ isotopes. The $2_{1}^{+}$energies and $B(E 2)$ values were obtained in Hartree-Fock-Bogolyubov based configuration mixing calculations using the the Gogny D1S force $[35,36]$ and the generator coordinate method with Gaussian overlap approximation, treating axial and triaxial quadrupole deformations [37]. The calculations use a triaxial harmonic oscillator basis including 11 major shells. The collective masses are calculated as described in Ref. [38]. Albeit the $2_{1}^{+}$energies are on average $30 \%$ too high, the systematic trend is reasonably well reproduced, considering that the model contains no free parameters (and no effective charges). The calculated $B(E 2)$ values show only a small increase at $N=40$, which can also be attributed to the occupancy of the $\nu g_{9 / 2}$ orbital. This occupancy is found to increase from approximately 0.6 in in the ground state of ${ }^{60} \mathrm{Fe}$ to 2.5 in ${ }^{64} \mathrm{Fe}$ [39]. The occupancy of the $d_{5 / 2}$ orbital in the ground state of ${ }^{64} \mathrm{Fe}$ is found to be 0.06 , i.e. smaller than in the shell model. A more important difference, however, is the fact that the $N=40$ gap remains more or less constant in the Gogny mean-field calculation, performed at deformation zero, with $\Delta E \approx 4 \mathrm{MeV}$ from $Z=20$ to $Z=40$ (see Fig. 1 in Ref. [34]), whereas in the shell model the gap is strongly reduced for $Z<28$. Although it is not obvious that single-particle energies from shell model and spherical mean-field calculations can be directly compared, since the latter do not contain any correlations beyond the mean field, the different development of the $N=40$ shell gap could explain the more gradual increase of $B(E 2)$ values in the Gogny calculation. On the other hand, also proton excitations and the size of the $Z=28$ gap could contribute to the difference in collectivity. More theoretical work is needed to understand the origin of the differences between the shell model and mean-field predictions. While experimental data on ${ }^{60} \mathrm{Ca}$ remain out of reach with present-day techniques, the measurement of $B(E 2)$ values in neutron-rich $\mathrm{Cr}$ isotopes will already shed more light on this question and allow making better predictions for a possible doubly-magic nature of ${ }^{60} \mathrm{Ca}$.

In summary, the lifetimes of the $2_{1}^{+}$states in ${ }^{62} \mathrm{Fe}$ and ${ }^{64} \mathrm{Fe}$ have been measured using the RDDS technique in combination with multi-nucleon transfer reactions in inverse kinematics. A steep increase of the $B\left(E 2 ; 2_{1}^{+} \rightarrow 0_{1}^{+}\right)$value is observed from ${ }^{62} \mathrm{Fe}$ to ${ }^{64} \mathrm{Fe}$. The comparison with both shell model calculations and a microscopic collective model based on the Gogny force allows relating the increase in collectivity to the occupation of neutron intruder orbitals. The mechanism causing this onset of collectivity below ${ }^{68} \mathrm{Ni}$ is found to be similar to the one responsible for the island of inversion around ${ }^{32} \mathrm{Mg}$. The two theoretical approaches predict a different structure of the more exotic nuclei with $N \approx 40$.

The authors would like to thank the technical staff at GANIL for excellent support and A. Navin for fruitful discussions. This work was partly supported by DFG (Germany) under Contract No. DE1516/-1.
[1] O. Sorlin, M.-G. Porquet , Prog. Part. Nucl. Phys. 61, 602 (2008).

[2] T. Otsuka, T. Suzuki, R. Fujimoto, H. Grawe, Y. Akaishi, Phys. Rev. Lett. 95, 232502 (2005).

[3] R. Broda et al., Phys. Rev. Lett. 74, 868 (1995).

[4] O. Sorlin et al., Phys. Rev. Lett. 88, 092501 (2002).

[5] C. Guénaut et al. Phys. Rev. C 75, 044303 (2007).

[6] S. Rahaman et al., Eur. Phys. J. A 34, 5 (2007).

[7] K. Langanke, J. Terasaki, F. Nowacki, D.J. Dean,
W. Nazarewicz, Phys. Rev. C 67, 044314 (2003).

[8] J. Van de Walle et al., Phys. Rev. Lett. 99, 142501 (2007).

[9] E. Padilla-Rodal et al., Phys. Rev. Lett. 94, 122501 (2005).

[10] M. Hannawald et al., Phys. Rev. Lett. 82, 1391 (1999).

[11] P. Adrich et al., Phys. Rev. C 77, 054306 (2008).

[12] O. Sorlin et al., Eur. Phys. J. A 16, 55 (2003).

[13] N. Aoi et al., Phys. Rev. Lett. 102, 012502 (2009). 
[14] K. Kaneko, Y. Sun, M. Hasegawa, T. Mizusaki, Phys. Rev. C 78, 064312 (2008).

[15] N. Hoteling et al., Phys. Rev. C 74, 064313 (2006).

[16] S. Lunardi et al., Phys. Rev. C 76, 034303 (2007).

[17] M. Block et al., Phys. Rev. Lett. 100, 132501 (2008).

[18] J.J. Valiente-Dobón et al., Phys. Rev. C 78, 024302 (2008).

[19] H.L. Crawford et al., Phys. Rev. C 79, 054320 (2009).

[20] S.J. Freeman et al., Phys. Rev. C 69, 064301 (2004).

[21] A. Bürger et al., Phys. Lett. B622, 29 (2005).

[22] E.K. Warburton, J.W. Olness, A.M. Nathan, J.J. Kolata, J.B. McGrory, Phys. Rev. C 16, 1027 (1977).

[23] O. Perru et al., Phys. Rev. Lett. 96, 232501 (2006).

[24] A. Dewald et al., Z. Phys. A 334, 163 (1989).

[25] J.J. Valiente-Dobón et al., Phys. Rev. Lett. 102, 242502 (2009).

[26] S. Pullanhiotan et al., Nucl. Instr. Meth. A 593, 343 (2008).
[27] J. Simpson et al. Acta Phys. Hung. N. S. 11, 159 (2000).

[28] T. Pissulla, A. Dewald et al., Nucl. Instr. Meth. A, to be published.

[29] S. Raman et al., At. Data Nucl. Data Tables 78, 1 (2001).

[30] E. Caurier et al., Eur. Phys. J. A 15, 145 (2002).

[31] M. Hjorth-Jensen, T.T.S. Kuo, E. Osnes, Phys. Rep. 261, 125 (1995).

[32] S.M. Lenzi et al. to be published

[33] A.P. Zuker, J. Retamosa, A. Poves, E. Caurier, Phys. Rev. C 52, R1741 (1995).

[34] L. Gaudefroy et al., Phys. Rev. C 80, 064313 (2009).

[35] J. Dechargé, D. Gogny, Phys. Rev. C 21, 1568 (1980).

[36] J.-F. Berger, M. Girod, D. Gogny, Comput. Phys. Commun. 63, 365 (1991).

[37] J.-P. Delaroche et al., Phys. Rev. C 81, 014303 (2010).

[38] M. Zielińska et al., Phys. Rev. C 80, 014317 (2009).

[39] J.M. Daugas et al., to be published. 